\title{
Studies of OFDM Signal for Broadband Optical Access Networks
}

\author{
C. W. Chow, Member, IEEE, C. H. Yeh, C. H. Wang, Student Member, IEEE, C. L. Wu, S. Chi, and \\ Chinlon Lin, Fellow, IEEE
}

\begin{abstract}
Using orthogonal-frequency-division-multiplexing (OFDM) format for passive optical network (PON) is a subject of great interest for recent research works. OFDM signal has high spectral efficiency, high tolerance to the fiber chromatic dispersion and the high flexibility on both multiple services provisioning and dynamic bandwidth allocation. In this paper we study the use of OFDM signals for optical access networks, including carrier distributed PON, heterogeneous optical wired/wireless network and a $100 \mathrm{~Gb} / \mathrm{s}$ OFDM-PON. We first quantify the performance of the OFDM signal when subjected to the noise produced by two different Rayleigh backscattering (RB) components that are present in the carrier-distributed PON. Then, based on these results, we also study the possibility of using OFDM for signal remodulation in a long-reach (LR)-PON. We propose carrier-distributed OFDM PONs using a dual-feeder fiber architecture and wavelength-shifting to mitigate the RB noise. Afterwards, we propose heterogeneous optical wired/wireless access networks, combining $10 \mathrm{~Gb} / \mathrm{s}$ PON and $10 \mathrm{~Gb} / \mathrm{s}$ OFDM radio-over-fiber (ROF) network. Finally, we propose a $100 \mathrm{~Gb} / \mathrm{s}$ OFDM-PON using subcarrier multiplexing (SCM).
\end{abstract}

Index Terms-passive optical networks (PONs), orthogonal frequency division multiplex (OFDM), Rayleigh backscattering, heterogeneous wired/wireless network.

\section{INTRODUCTION}

A STRONG upgrade of existing access network is required in order to cope with the exponential increase of bandwidth demand. The capacities of next generation access networks will be supporting $10 \mathrm{~Gb} / \mathrm{s}$ and $40 \mathrm{~Gb} / \mathrm{s}$. Passive optical network (PON) is a promising candidate for these high capacity broadband optical access networks due to its cost effectiveness [1]. However, unlike long haul optical networks, access networks are very cost-sensitive. The optical networking units (ONUs) employed in customer apartments should be simple and low cost. Orthogonal frequency division multiplexed (OFDM) signal has been proposed to be deployed in long-haul optical networks due to its robustness against fiber chromatic dispersion [2-6]. Recently, OFDM signal has also shown its usefulness for next-generation PON [7-13] and long reach (LR) hybrid wavelength division multiplexed (WDM)/

Manuscript received 15 August 2008; revised 20 January 2010. This work was supported by the National Science Council, Taiwan, R.O.C., under Contract NSC-96-2218-E-009-025-MY2, NSC-97-2221-E-009-038-MY3, NSC98-2221-E-009-017-MY3C.

C. W. Chow, C. H. Wang, C. L. Wu, S. Chi, and C. Lin are with the Department of Photonics, Institute of Electro-Optical Engineering, National Chiao Tung University, Hsinchu, Taiwan (e-mail: cwchow@faculty.nctu.edu.tw).

C. H. Yeh is with the Information and Communications Research Laboratories, Industrial Technology Research Institute, Taiwan. Chinlon Lin is also with the School of EEE, Nanyang Technological University, Singapore.

Digital Object Identifier 10.1109/JSAC.2010.100805. time division multiplexed (TDM) PON [14-15]. Due to its high spectral efficiency, low cost and low bandwidth optical components can be used. It has a strong tolerance to the fiber chromatic dispersion, and this feature is very important to PON and LR-PON since these networks usually cannot be fully dispersion compensated. It has high flexibility on both multiple services provisioning and dynamic bandwidth allocation, allowing easy bandwidth management in different ONUs.

The basic concept of OFDM is that data is transmitted on a number of different carrier frequencies in parallel; hence, the total data rate is divided by the number of frequency channels used in the system. Since the symbol period is much longer than that of the original serial data having the same total data rate, inter-symbol interference (ISI) can be effectively mitigated. Besides, the residual ISI can be removed by using the cyclic prefix (CP), a guard time interval used in most of the OFDM system. The OFDM frequency diversity transmission enables equalization of frequency response by baseband digital signal processing (DSP). And it also offers the prospect of integrating forward error correction (FEC) to improve transmission.

WDM-PON has been considered to increase the network capacity by using multiple wavelength channels. The technology challenge for WDM-PON is to avoid the need for expensive wavelength tunable optical elements in each ONU. In practice this means that it is cost prohibitive to use the type of lasers currently available for long haul WDM transmission within a WDM-PON. Moreover, it would be impractical for each customer ONU to be built with a fixed single wavelength laser because managing the inventory of lasers would be complex and costly for the network operators. Hence, customer ONU should be "colorless", working in reflective mode and generating the upstream signal using the optical carriers distributed from the optical line terminal (OLT) in the head-end office. Previous works showed that carrier distributed OFDM-PON suffers form interferometric beat noise generated by Rayleigh backscattering (RB) [7]. However, the research and characterization of the OFDM signal when subjected to the RB noise in carrier distributed PON is missing. Here, we first quantify the performance of the OFDM signal when subjected to the noise generated by the two different components of RB that are present in the carrier distributed PON. Then, based on these results, we also study the possibility of using OFDM for signal remodulation in the LR-PON. OFDM signal is spectral efficient and has high chromatic dispersion tolerance. In this work, we studied the 

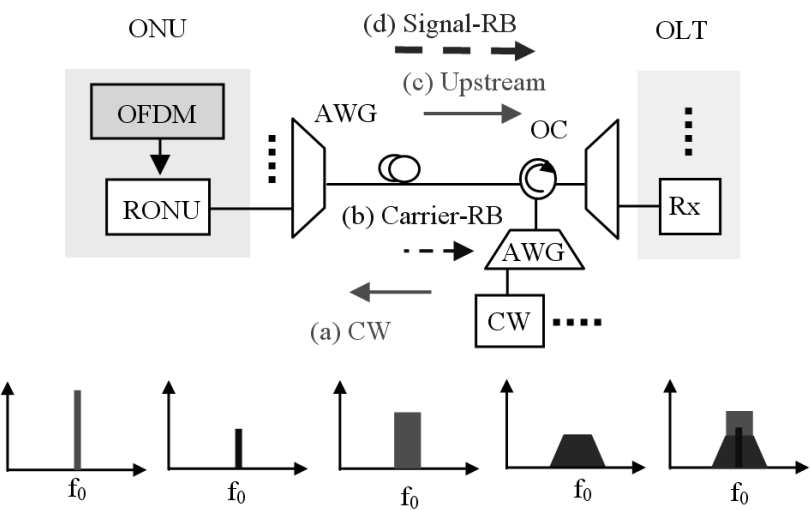

(a) CW

(b) Carrier-RB

(c) Upstream

(d) Signal-RB

(e) Total

Fig. 1. Schematic of carrier distributed PON. AWG: arrayed waveguide grating, OC: optical circulator. Insets: schematic optical spectra at different locations.

possibility of using OFDM signal in several different optical network architectures. We propose carrier distributed OFDM PONs using dual-feeder fiber and using wavelength-shifting to mitigate $\mathrm{RB}$ noise.

After this, we will propose heterogeneous optical wired/wireless access networks, combining $10 \mathrm{~Gb} / \mathrm{s}$ PON and $10 \mathrm{~Gb} / \mathrm{s}$ OFDM radio-over-fiber (ROF) network. Heterogeneous wired/wireless access network has been proposed to integrate the advantages of optical wired and wireless access networks, providing high capacity and flexible broadband connections for both fixed and mobile users [16]. Finally, we will propose a $100 \mathrm{~Gb} / \mathrm{s}$ OFDM-PON using subcarrier multiplexing (SCM), without the need of using polarization multiplexing [4], WDM [17] nor optical comb generator [18].

The paper is organized as follows: In Section II, we will analyze the RB of OFDM signal in a carrier distributed PON. In Section III, we will demonstrate a carrier distributed OFDM LR-PON using dual-feeder fiber architecture to mitigate RB noise. A RB mitigation scheme using wavelength-shifting will also be proposed. In Section IV, we will propose the heterogeneous wired/wireless access networks. Then in Section V, we will propose and demonstrated using numerical simulation a $100 \mathrm{~Gb} / \mathrm{s}$ OFDM-PON using SCM. A conclusion will be given in Section VI.

\section{RAYLEIGH BACKSCATTERING ANALYSIS OF OFDM} SIGNAL

Carrier distributed WDM-PONs provide many attractive features, however, when a single drop fiber is employed to reach the customer, the carrier distributed from the OLT and the upstream signal must share the same path, giving rise to interferometric beat noise caused by the RB. RB is generated by the distributed reflections caused by the random index fluctuation along the silica optical fiber. The RB noise is partially polarized in nature, with a colored power spectral density (PSD) proportional to the PSD of the input signal to the optical fiber. We identified two main contributions to the RB noise, as shown in Fig. 1 in the carrier distributed PONs. They interfere with the upstream signal at the receiver (Rx). The first contribution, Carrier-RB, is generated by the

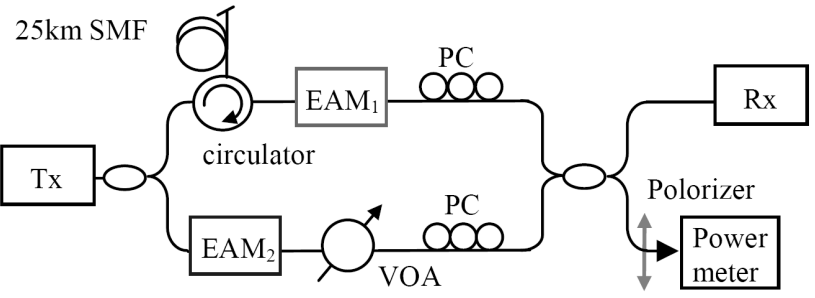

Fig. 2. Experimental setup for OCSR analysis. VOA: variable optical attenuator, EAM: electro-absorption modulator.

backscatter of the continuous wave $(\mathrm{CW})$ carrier delivered to the reflective ONU (RONU). The second contribution, Signal$\mathrm{RB}$, is generated by the upstream data signal at the output of the RONU. Backscattered light from this upstream signal reenters the RONU, and it is modulated at a second time (hence, having a broader spectrum) and transmitted towards the Rx.

As mentioned before, the RB noise is partially polarized in nature, with a colored PSD proportional to the PSD of the input signal. As a result, the spectra of Carrier-RB and the $\mathrm{CW}$ carrier are nearly the same, while the Signal-RB is modulated twice by the RONU and has a much broader spectrum. The relative impact of the two components depends on the exact network configuration and hence, for a full understanding, separate analysis of each effect is needed. Insets of Fig. 1 indicate the schematic optical spectra at different locations of a carrier distributed PON. Higher optical spectral overlapping will produce higher electrical beat noise at the same frequency band when detected by a photodetector. A more accurate approach is to use the PSD of each signal, and the modeling has been described in detail in [19].

A theoretical derivation of the RB properties of a signal has been successfully achieved recently [19]. Here, we experimental characterize the optical crosstalk to signal ratio (OCSR) performance of the upstream signal subjected to Carrier-RB and Signal-RB, as shown in Fig. 2. The OCSR is defined as the power ratio of $\mathrm{RB}$ crosstalk noise to the data signal measured just prior the Rx. For the two cases, the signals were modulated at different points in the setup of Fig. 2 to reproduce the modulation characteristics of a carrier distributed PON and to allow separate analysis of the two RB components. For the Carrier-RB analysis, the transmitter (Tx) in Fig. 2 was a CW signal $(1548 \mathrm{~nm}$, output power $6 \mathrm{dBm})$. It was split by a 3-dB fiber coupler between two output paths. The upper path was used to generate $\mathrm{CW}$ Carrier-RB and hence $\mathrm{EAM}_{1}$ was not used. The Carrier-RB produced by a $25 \mathrm{~km}$ single mode fiber (SMF), terminated with an angled connector, was extracted via an optical circulator (OC). In the lower path, the remainder of the $\mathrm{CW}$ was modulated by $\mathrm{EAM}_{2}$ to produce the data signal for analysis. A variable optical attenuator (VOA) was used to adjust the data signal power to generate different OCSRs. The Carrier-RB and the data signal were then co-polarized (the highest beat noise case) by using the polarization controllers (PCs). They were combined by the fiber coupler, and then launched into an optically pre-amplified $\mathrm{Rx}$ (formed by an erbium doped fiber amplifier (EDFA), an optical filter (Gaussian shaped, 3-dB bandwidth of $50 \mathrm{GHz}$ ) and a PIN photodiode) for bit-error rate (BER) measurements. 


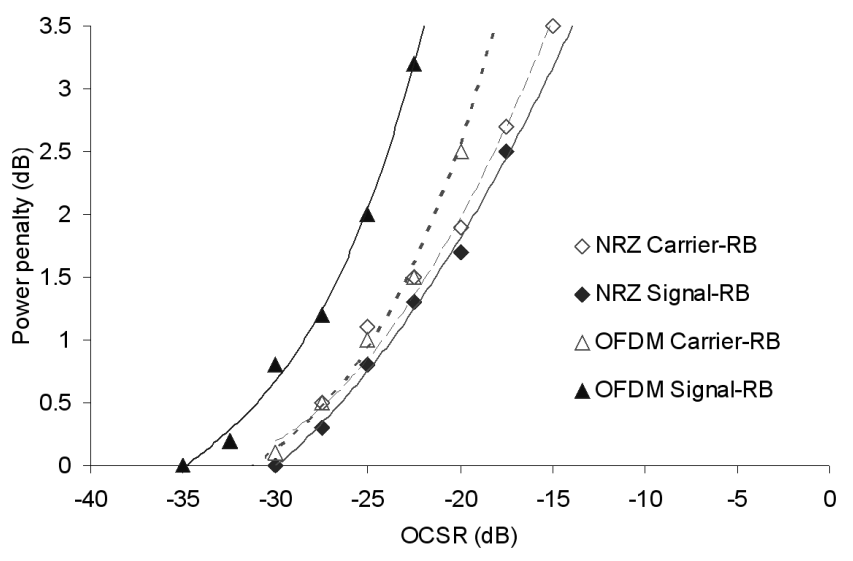

Fig. 3. RB noise performance of OFDM signal when compared with conventional NRZ formats.

In the Signal-RB analysis, the Tx consisted of a CW laser and an EAM to produce the data signal. In the upper path, the backscattered data signal by the $25 \mathrm{~km}$ SMF was modulated again by $\mathrm{EAM}_{1}$ after the OC to generate the Signal-RB. In the lower path, $\mathrm{EAM}_{2}$ was unused. The Signal-RB and the data signal were then combined before being launched into the Rx.

The OFDM signal was generated by a digital-to-analog converter (DAC), which is an arbitrary waveform generator with $4 \mathrm{GHz}$ sampling rate, as described in [15]. The data was packed into 16 subcarriers, each subcarrier symbol was a 16-quadrature amplitude modulation (QAM) format. The OFDM signal occupied a bandwidth of $1 \mathrm{GHz}(62.5 \mathrm{MHz}$ to $1.125 \mathrm{GHz}$ ), and the total data rate of the OFDM signal was $4 \mathrm{~Gb} / \mathrm{s}$. By using inverse fast Fourier transform (IFFT), these subcarrier symbols were converted to a time-domain OFDM symbol. They were applied to the EAMs in Fig. 2 with proper bias. The OFDM signal was directly detected by the Rx. An analog-to-digital converter (ADC), which is a realtime oscilloscope, connected to the Rx converted the OFDM signal to digital format. Synchronization of extracted carrier phase and fast Fourier transform (FFT) for time to frequencydomain translation was performed using off-line computer software. Then the QAM decoder analyzed the symbol on each subcarrier. The BER was calculated from the measured error vector magnitude (EVM). For comparison, the Carrier$\mathrm{RB}$ and Signal-RB of $4 \mathrm{~Gb} / \mathrm{s}$ non-return to zero (NRZ) signals were also evaluated using the similar setup shown in Fig. 2.

The RB performance of a signal depends on the interferometric beat noise falling within the $\mathrm{Rx}$ bandwidth. The interferometric beat noise depends on the spectral overlap between the data signal and the interferers. Fig. 3 shows the measured RB performances by comparing the power penalties at BER of $10^{-9}$, as a function of OCSRs. For conventional NRZ signal, the OCSR is about $-25 \mathrm{~dB}$ [20]. This means that when the $\mathrm{RB}$ noise power is $25 \mathrm{~dB}$ less than the data signal power, the power penalty is $\sim 1 \mathrm{~dB}$. The measurement result shows that the Carrier-RB performance of OFDM-QAM is better than its Signal-RB performance. This is due to the fact that Carrier-RB beat noise is low frequency in nature, and the OFDM signal is slightly up-converted $(62.5 \mathrm{MHz}$ to $1.125 \mathrm{GHz}$ ), hence, the Carrier-RB noise mainly falls within the frequency gap between the center carrier and the OFDM

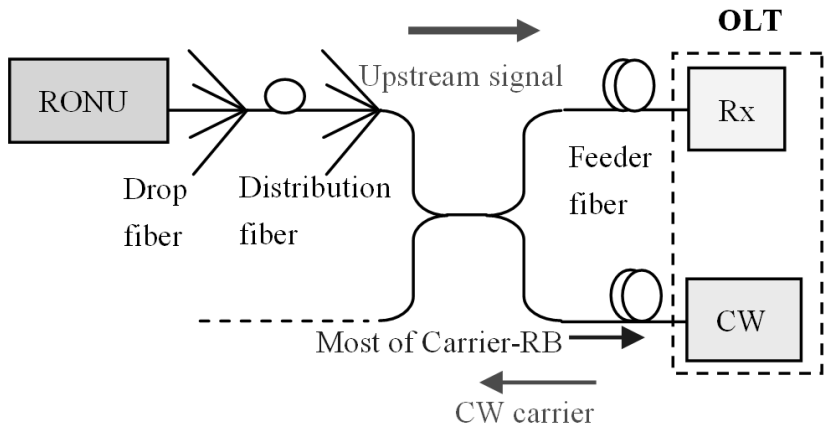

Fig. 4. Schematic of a carrier distributed PON using dual-feeder fiber architecture.

signal. The Signal-RB has a wider spectrum than the OFDM signal since it has been modulated twice. And the noise spectrum of the Signal-RB will occupy the whole frequency band of the OFDM signal, producing much higher beat noise due to the complete spectral overlap. As a result, we can observe from Fig. 3 that the Signal-RB OCSR of the OFDM signal is $-30 \mathrm{~dB}$ at $1-\mathrm{dB}$ power penalty window. The $\mathrm{RB}$ performance of NRZ signal was also included. Results indicate that OFDM signal has similar Carrier-RB performance to NRZ signal, but poorer Signal-RB performance than NRZ signal.

\section{OFDM SIGNAL REMODULATION LR-PON}

Signal remodulation could be one of the promising candidates for next generation PONs [11-12, 21-23]. Expensive Tx inside the ONU with distinct and specific wavelength has hindered the PON deployment. Network architectures with signal remodulation on the downstream channel for the upstream channel can reduce the cost of wavelength referencing, control and stabilization at the cost-sensitive ONU. Only a single wavelength is needed for both downstream and upstream signals, since wavelength is reused.

According to the OCSR analysis described in Section II, the RB noise can degrade the performance of a carrier distributed PON using OFDM signal. And the noise generated by the Signal-RB is worse than that of NRZ signal. Here, we study the possibility of using OFDM format for signal remodulation LR-PON. We also propose and demonstrate using dual-feeder fiber architecture to mitigate the RB noise in the OFDM signal remodulation LR-PON. Colorless RONU based on reflective semiconductor optical amplifier (RSOA) and Fabry-Perot laser diode (FP-LD) are evaluated and compared.

If we consider a carrier-distributed PON with two stages of splitters such as the one shown in Fig. 4, where for clarity the arrayed waveguide grating (AWGs) are not shown, we can see clearly that most of the Carrier-RB power is generated by the optical carrier in the fiber (feeder fiber) before the first optical splitter. This is due to the loss introduced by the first optical splitter and also because the feeder fiber usually have most of the fiber length. The Carrier-RB generated before the first splitter cannot reach the Rx and thus it cannot interfere with the upstream signal. Although the Carrier-RB generated in the distribution and drop sections, and the Signal-RB still propagates to the $\mathrm{Rx}$, the overall $\mathrm{RB}$ power is significantly reduced compared with a system using a single feeder fiber. 


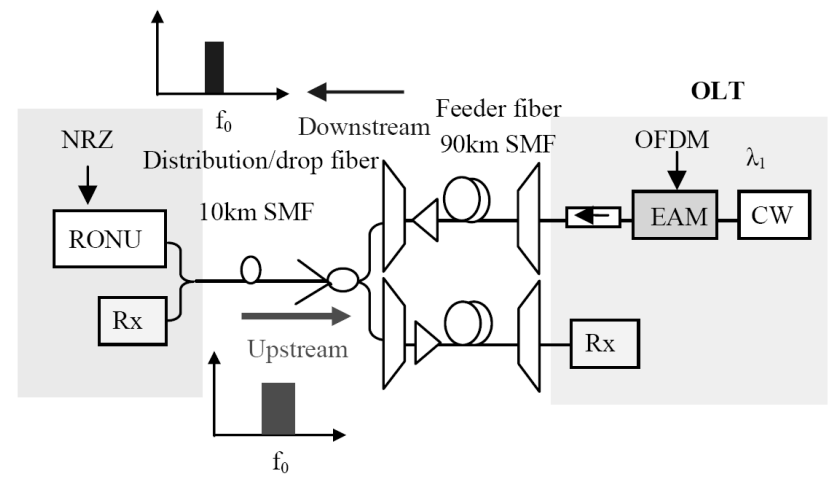

Fig. 5. Experimental setup of signal remodulation LR-PON. EAM: electroabsorption modulator, SMF: single mode fiber.

Besides, The circulator used to launch the carrier in singlefeeder scheme is not needed in the dual-feeder architecture, since the optical splitter used to combine the two feeder fibers performs this function. Detail analysis of using dual-feeder fiber architecture can be found in [24]. This scheme also maintains the benefit of a single drop and distribution fiber to each ONU, which could reduce the cost of fiber and fiber connections in the network [24].

Fig. 5 shows the experimental setup of a signal remodulation LR-PON. The downstream electrical OFDM signal (as described in Section II) was applied to the EAM. The signal was then transmitted via $90 \mathrm{~km}$ feeder and $10 \mathrm{~km}$ distribution/drop fibers before being launched into the RONU. At the RONU, $10 \%$ of downstream power was tapped out and detected by an optically pre-amplified Rx. $90 \%$ of the downstream signal was then launched into the colorless modulator at the ONU. Two different kinds of colorless modulators: RSOA and FP$\mathrm{LD}$ are used. In each case, a $2.5 \mathrm{~Gb} / \mathrm{s} \mathrm{NRZ}$ data, pseudo random binary sequence (PRBS) pattern length of $2^{31}-1$, was used to directly modulate the colorless modulator.

We have studied that when the extinction ratio (ER) of the downstream OFDM signal was $\sim 4 \mathrm{~dB}$, the RONU can effectively suppress the ER of the downstream OFDM signal by using the gain-saturation property of the RSOA [25] or the injection locking property of the FP-LD [26]. Fig. 6 shows the BER measurements of each signal at back-to-back (b2b) and after the $100 \mathrm{~km}$ LR transmission. Insets show the constellation diagrams of OFDM signal at ER $\sim 4 \mathrm{~dB}$, output remodulated NRZ eye diagrams of FP-LD and RSOA when downstream OFDM at ER $\sim 4 \mathrm{~dB}$. We can observe clear open eyes after $100 \mathrm{~km}$ SMF transmission without dispersion compensation in each case. Error-free BER operation can be achieved for the proposed remodulation LR system. We also studied the nonlinear transmission characteristics of the RSOA- and FP-LD-based colorless RONUs. Different CW optical powers were launched into them at different biases, and we measured their output optical powers using power meter. For the injection locked FP-LD-based RONU, when the FP-LD was biased below the threshold current $(10 \mathrm{~mA})$, the transfer characteristic was quite linear. When the biased current was much above threshold (35 mA), the nonlinear transfer function was much steeper than that of the RSOAbased RONU; and there was an abrupt output power change

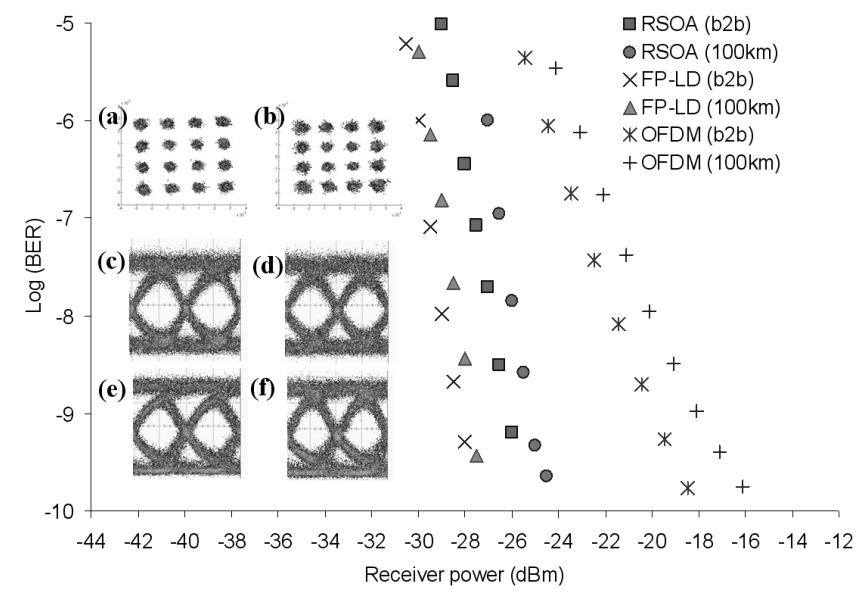

Fig. 6. BER measurements of different signals. Insets: constellation diagrams of OFDM signal at ER $\sim 4 \mathrm{~dB}$ at (a) b2b, (b) $100 \mathrm{~km}$, output remodulated NRZ eye diagrams of FP-LD at (c) b2b, (d) $100 \mathrm{~km}$; and RSOA at (e) b2b, (f) $100 \mathrm{~km}$, when downstream OFDM at ER $\sim 4 \mathrm{~dB}$.

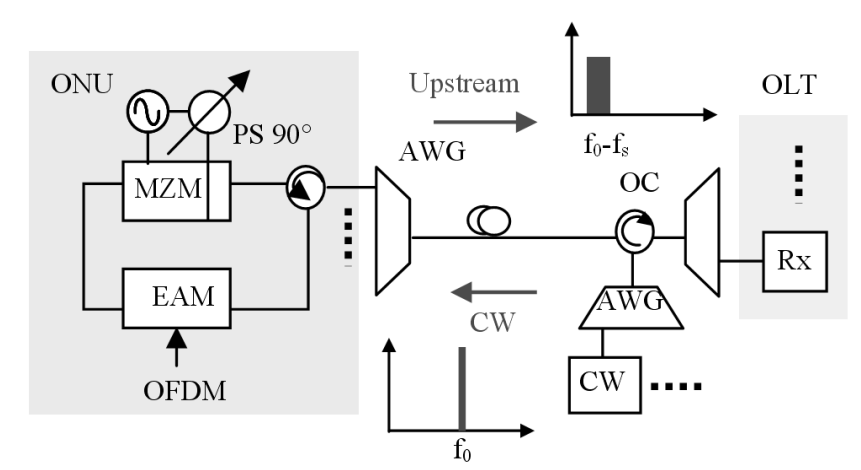

Fig. 7. Schematic of carrier distributed OFDM-PON using wavelengthshifting. OC: optical circulator, PS: phase shifter, MZM: dual-arm MachZehnder Modulator, EAM: electro-absorption modulator.

owing to the injection locking. Hence, the downstream OFDM signal can be highly suppressed by the injection locking of the FP-LD-based RONU, producing a better upstream NRZ signal. We can observe in Fig. 6 that the BER performance of FP-LD remodulation is better than the RSOA remodulation. The disadvantage of using FP-LD is that it is sensitive to the input polarization, and polarization tracking is necessary. It is possible to use NRZ signals for both upstream and downstream. It is worth to note that although one wavelength was demonstrated, WDM is also supported.

We also proposed and demonstrated by simulation (VPI Transmission Maker V7.5) another RB mitigation scheme in the carrier distributed OFDM-PON without using the dualfeeder architecture. Fig. 7 shows the schematic of the carrier distributed OFDM-PON, where wavelength-shifting was used to mitigate RB. The CW carrier $\left(f_{0}\right)$ was launched towards the colorless ONU. A dual-arm MZM inside the ONU was driven in-phase and quadrature-phase by an electrical sinusoidal signal at frequency of $10 \mathrm{GHz}\left(f_{s}\right)$ to produce the single-sideband carrier suppressed (SSB-CS) or a wavelength-shifted $\left(f_{0}-f_{s}\right)$ signal. The signal was then encoded by the OFDM signal (the generation method was described in previous paragraph) and then sent back towards the Rx at OLT. 


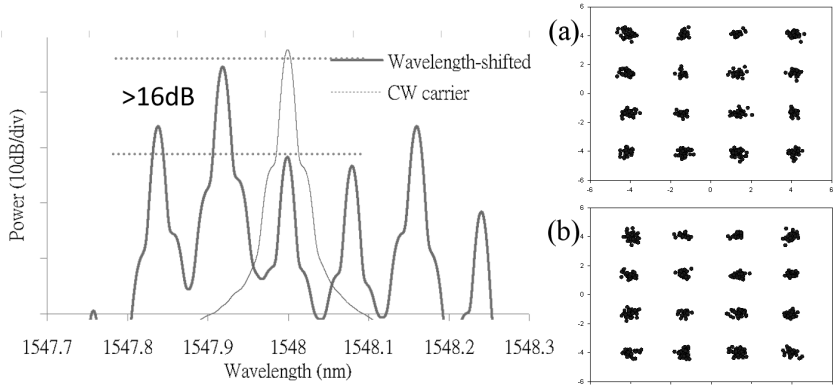

Fig. 8. Simulated optical spectra of the $\mathrm{CW}$ carrier and upstream signal. Insets: Simulated constellation diagrams of upstream signal at BER of $10^{-9}$ when (a) without wavelength-shifting and OCSR $=-25 \mathrm{~dB}$, (b) with wavelength-shifting and OCSR $=-15 \mathrm{~dB}$.

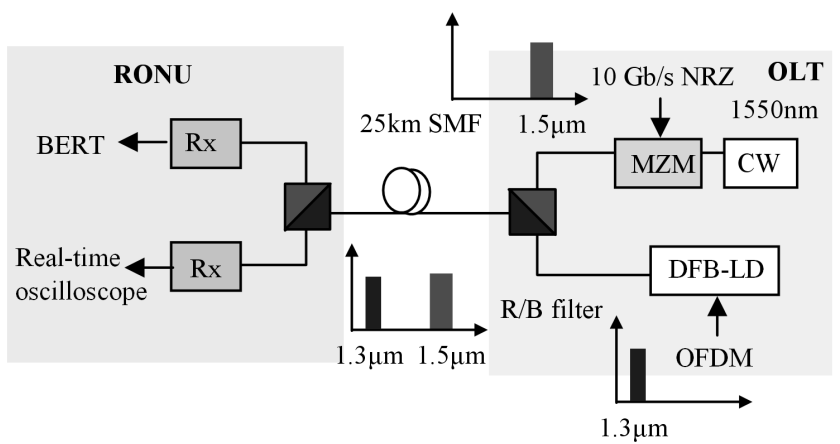

Fig. 9. Experimental setup of the heterogeneous $10 \mathrm{~Gb} / \mathrm{s}$ PON and $10 \mathrm{~Gb} / \mathrm{s}$ OFDM ROF network. DFB-LD: distributed-feedback laser diode, R/B filter: red/blue filter, SMF: single mode fiber.

Fig. 8 shows the simulated optical spectra of the distributed $\mathrm{CW}$ carrier $\left(f_{0}\right)$ and the wavelength-shifted OFDM signal $\left(f_{0}-f_{s}\right)$. The power ratios between the upper sideband to the lower sideband and the center wavelength are $>16 \mathrm{~dB}$. Since the RB tolerance depends on the interferometric beat noise falling within the Rx bandwidth, the RB tolerance of the wavelength-shifted signal can be improved due to the reduced spectral overlap with the carrier wavelength. Inset of Fig. 8(a) and (b) show the simulated constellation diagrams of upstream 16-QAM OFDM signal at the same BER of $10^{-9}$ at the 1-dB power penalty window (Fig. 3) without wavelength-shifting $(\mathrm{OCSR}=-25 \mathrm{~dB})$ and with wavelength-shifting $(\mathrm{OCSR}=$ $15 \mathrm{~dB}$ ) respectively. Result show that wavelength-shifting can significantly mitigate RB generated by the $\mathrm{CW}$ carrier by withstanding a much higher RB noise (OCSR improved by $10 \mathrm{~dB})$.

\section{HETEROGENEOUS OPTICAL WIRED/WIRELESS NETWORK}

Here we demonstrate a fully passive (without optical amplifier in the transmission link) heterogeneous optical wired/wireless access network, combining $10 \mathrm{~Gb} / \mathrm{s}$ PON and $10 \mathrm{~Gb} / \mathrm{s}$ OFDM ROF network. OFDM cannot only be applied as baseband signal in PON, but also be used in the present radio-frequency (RF) communications. OFDM is used here since it is commonly used in the present wireless communication. Fig. 9 shows the experimental setup of the proposed heterogeneous $10 \mathrm{~Gb} / \mathrm{s}$ PON and $10 \mathrm{~Gb} / \mathrm{s}$ OFDM ROF network. At the OLT,
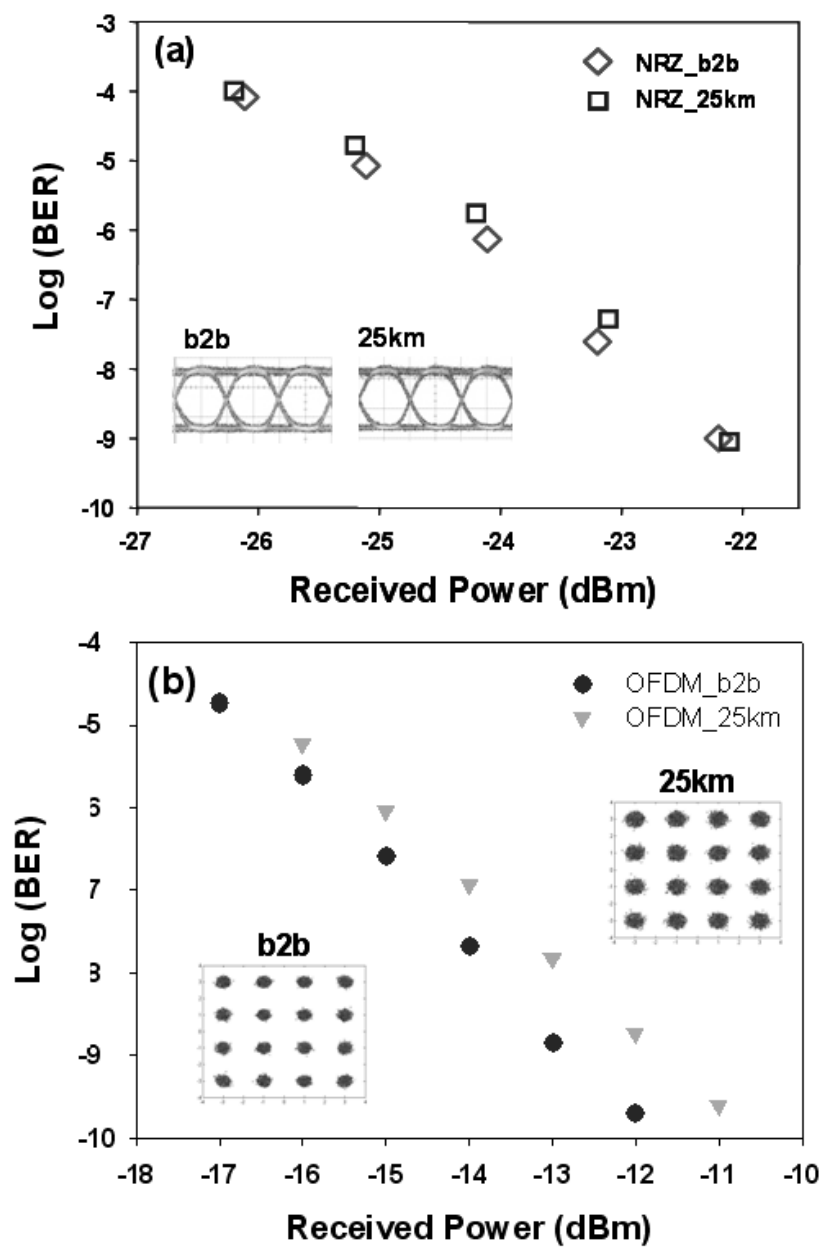

Fig. 10. BER measurements of (a) $10 \mathrm{~Gb} / \mathrm{s}$ NRZ and (b) $10 \mathrm{~Gb} / \mathrm{s}$ OFDM signals at back-to-back (b2b) and after $25 \mathrm{~km}$ SMF transmission. Insets: corresponding NRZ eye diagrams and OFDM constellation diagrams.

the $10 \mathrm{~Gb} / \mathrm{s}$ NRZ data was used to broadcast the downstream information for wired applications and it was operated at 1550 $\mathrm{nm}$. The $10 \mathrm{~Gb} / \mathrm{s}, 2^{31}-1$ PRBS NRZ optical signal was encoded by using a Mach-Zehnder modulator (MZM).

For the ROF part, the total $10 \mathrm{~Gb} / \mathrm{s}$ OFDM-QAM signal was generated using similar method as described in Section II. Here, the arbitrary waveform generator has the $50 \mathrm{GHz}$ sampling rate. The incoming bit streams were packed into 128 subcarrier symbols, each subcarrier symbol was in 16-QAM format. In this experiment, the OFDM signal was directly modulated at a distributed feedback laser diode (DFB-LD) module of wavelength at $1312 \mathrm{~nm}$ with an average optical output power of $5 \mathrm{dBm}$. Using OFDM at wavelength $1312 \mathrm{~nm}$ was due to the available of a directly modulation (modulation bandwidth $>3 \mathrm{GHz}$ ) DFB-LD at laboratory. Due to the highly spectral efficiency of the QAM used in the OFDM signal, the total $10 \mathrm{~Gb} / \mathrm{s}$ OFDM data signal only occupied the bandwidth of $2.5 \mathrm{GHz}$. The OFDM signal was up-converted to a center frequency of $1.35 \mathrm{GHz}$.

Then, the red/blue filter (R/B filter) was used to combine the $1312 \mathrm{~nm}$ and $1550 \mathrm{~nm}$ signals together. The signal was then launched into a $25 \mathrm{~km}$ standard SMF without dispersion compensation. In the ONU, an optically pre-amplified Rx was used to detect the $10 \mathrm{~Gb} / \mathrm{s} \mathrm{NRZ} \mathrm{signal.} \mathrm{It} \mathrm{consisted} \mathrm{of} \mathrm{an}$ 


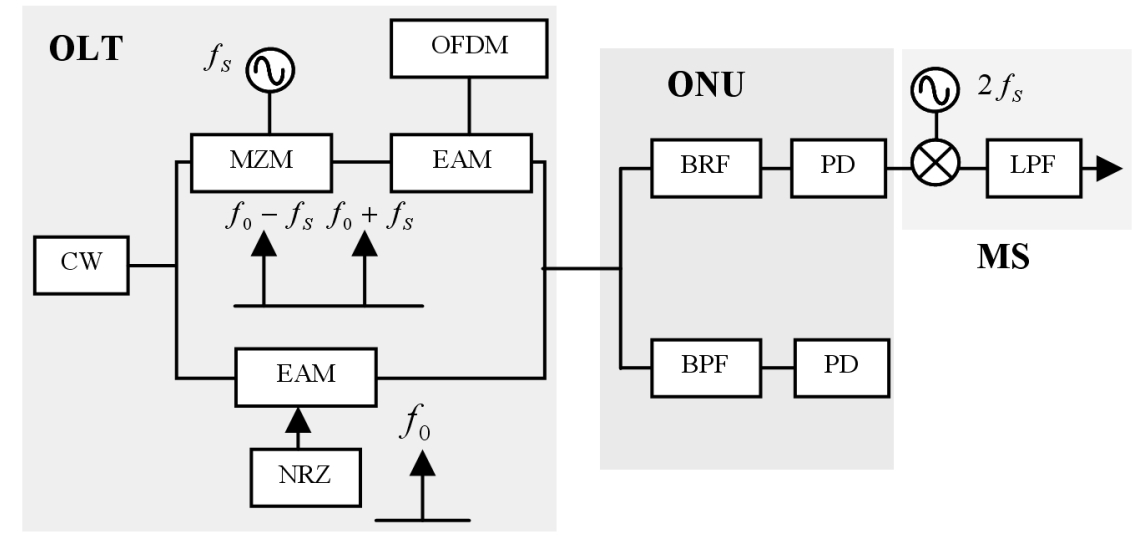

Fig. 11. Schematic of the heterogeneous network. MZM: Mach-Zehnder Modulator, EAM: electro-absorption modulator, BRF: band-rejection filter, BPF: bandpass filter, PD: photodiode, LPF: low pass filter.

EDFA with saturated output power of $12 \mathrm{dBm}$ and noise figure of $5 \mathrm{~dB}$, and an optical bandpass filter with 3-dB bandwidth of $50 \mathrm{GHz}$ to remove the out-of-band amplified spontaneous emission (ASE) noise, and a PIN photodiode. Due to the unavailable of the $1310 \mathrm{~nm}$ optical amplifier in the laboratory, only a PIN photodiode (without optical pre-amplifier) was used for the detection of OFDM signal.

In order to confirm the transmission performance of the proposed heterogeneous network, BER measurements were performed. Fig. 10(a) shows the BER measurements of the proposed $10 \mathrm{~Gb} / \mathrm{s}$ NRZ signal at b2b and transmission of 25 $\mathrm{km}$ SMF without dispersion compensation. We observed about $0.2 \mathrm{~dB}$ power penalty at BER of $10^{-9}$ in the transmission. Fig. 10(b) shows the BER measurements of $10 \mathrm{~Gb} / \mathrm{s}$ OFDM 16-QAM signals. About $1 \mathrm{~dB}$ power penalty was observed. Higher power penalty observed in the OFDM case was due to direct modulation of DFB-LD used. The requirement of relative larger received power than the NRZ signal is due to the unavailable of $1310 \mathrm{~nm}$ optical pre-amplifier. The corresponding NRZ eye diagrams and the OFDM constellation diagrams are shown in insets of Fig. 8.

We also proposed and demonstrated by simulation (VPI Transmission Maker V7.5) an integrated heterogeneous wired/wireless network as shown in Fig. 11. At the OLT, a CW signal was divided into two paths. An optical double-sideband carrier suppressed (DSB-CS) signal (separation between the two sidebands $=60 \mathrm{GHz}\left(=2 f_{s}\right)$ was produced by the MZM, which was electrical driven at $30 \mathrm{GHz}$ (fs) in the upper path. It was then encoded by $10 \mathrm{~Gb} / \mathrm{s}$ 16-QAM OFDM signal by the EAM. In the lower path, the CW was encoded by $10 \mathrm{~Gb} / \mathrm{s}$ NRZ signal by another EAM. The two signals were then combined at the output of the OLT producing the wired/wireless signal.

At the ONU, the signal was divided into two parts by a 3-dB fiber splitter. At the upper path of the ONU, an optical band rejection filter (BRF) with the rejection bandwidth of $20 \mathrm{GHz}$ was used to remove the NRZ signal at the center frequency (f0) for the wireless signal detection. By the coherent beating of the two sidebands of the DSB-CS OFDM signal at the photodiode (PD), an up-converted electrical OFDM signal at $60 \mathrm{GHz}$ carrier frequency was generated. Finally, the wireless signal was down-converted to baseband at the mobile station
(MS) by using a RF mixer, a $60 \mathrm{GHz}$ sinusoidal signal $\left(2 f_{s}\right)$ and a low pass filter (LPF) with bandwidth of $3 \mathrm{GHz}$. At the lower path, an optical bandpass filter (BPF) (Gaussian shaped, $20 \mathrm{GHz}$ bandwidth) was used to remove the wireless signal for the NRZ detection. We can observe that error-free transmissions can be achieved for both wired and wireless signals as shown in Fig. 12.

\section{100 G OFDM-PON USING SCM}

Finally in this Section, we will propose a $100 \mathrm{~Gb} / \mathrm{s}$ OFDMPON using SCM technique. Due to the unavailable of the high frequency RF mixers in the laboratory, the SCM channels were demonstrated by means of numerical simulation (using VPI Transmission Maker V7.5). Fig. 13 shows the schematic setup of the proposed $100 \mathrm{~Gb} / \mathrm{s}$ OFDM-PON. Ten channels (Ch 1 to $\mathrm{Ch} 10$ ) of OFDM signals were generated using the built-in modules in VPI. The bit streams were packed into 128 subcarrier symbols, and each subcarrier symbol was in 16-QAM format. Hence, each channel has a total data rate of $10 \mathrm{~Gb} / \mathrm{s}$, occupying a bandwidth of $2.5 \mathrm{GHz}$. Ch 2 to Ch 10 were up-converted by using RF mixers and RF clock sources, with frequency separation of $3 \mathrm{GHz}$. In order to improve the spectral efficiency, we added a electrical BPF (Bessel 3rd order, bandwidth of $3 \mathrm{GHz}$ ) after the mixer for each channel to remove one of the sideband of the RF up-converted signal, hence producing a single sideband (SSB) signal. Hence, the frequency separation between each SCM channel can be 3 GHz. They were then power combined and applied to a MZM with proper biases to encode the CW light source (1548 $\mathrm{nm}$ ) with the $100 \mathrm{~Gb} / \mathrm{s}$ OFDM signal. The signal was then launched into a Rx via $25 \mathrm{~km}$ of standard SMF and $4 \mathrm{~km}$ of dispersion compensating fiber $(\mathrm{DCF})$ (dispersion parameter $=$ $-100 \mathrm{ps} / \mathrm{nm} / \mathrm{km}$ ). The detected electrical signal was then power divided by using a wideband RF power splitter, and each SCM channel was demodulated and analyzed.

Fig. 14 shows the simulated BER curves of different individual SCM OFDM channels after the transmission. This means that there is only one active SCM channel in each simulation. We can observe that the power penalties for $\mathrm{Ch} 2$ to $\mathrm{Ch} 10$ are similar with variation of $\sim 1 \mathrm{~dB}$. Ch 1 performs better since this baseband channel has not been filtered by the BPF. 


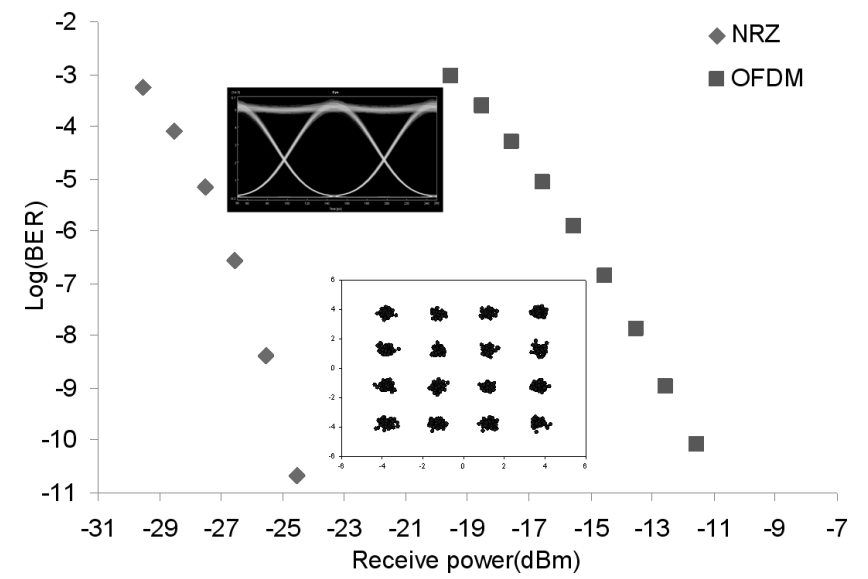

Fig. 12. BER measurements of $10 \mathrm{~Gb} / \mathrm{s} \mathrm{NRZ} \mathrm{and} \mathrm{down-converted} 10 \mathrm{~Gb} / \mathrm{s}$ OFDM signals at the Rx. Insets: corresponding NRZ eye diagrams and OFDM constellation diagrams.

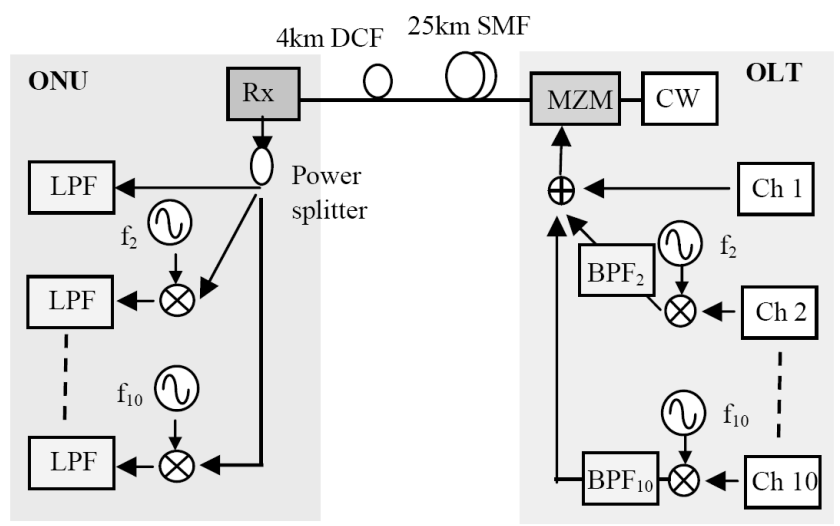

Fig. 13. Schematic setup of the $100 \mathrm{~Gb} / \mathrm{s}$ OFDM-PON using SCM. CW: continuous-wave light source, MZM: Mach-Zehnder modulator, LPF: low pass filter, BPF, bandpass filter, SMF: single mode fiber and DCF: dispersion compensating fiber.

Then we activated all the SCM channels, as shown in Fig. 15 , error free performance can still be observed in all SCM channels with higher power penalties when compared with Fig. 14. This is because by using SCM, the modulation index of each channel should be lowered to above clipping of the overall signal. We also observed that the power penalty increases with the channel number.

\section{CONCLUSION}

Using OFDM signal for PON is a subject of many research works recently. Previous researches show that carrier distributed OFDM-PON suffers form interferometric beat noise generated by RB. We first quantified the performance of OFDM signal when subjected to the noise generated by the Carrier-RB and Signal-RB that are present in the carrier distributed PON. The result shows that the Carrier-RB performance of OFDM-QAM is better than its Signal-RB performance. The Carrier-RB performance of OFDM-QAM signal is similar to that of NRZ signal, while the Signal-RB performance of the OFDM-QAM is worse than that of NRZ signal. Base on these results, we proposed and demonstrated an OFDM signal remodulation LR-PON using dual-feeder

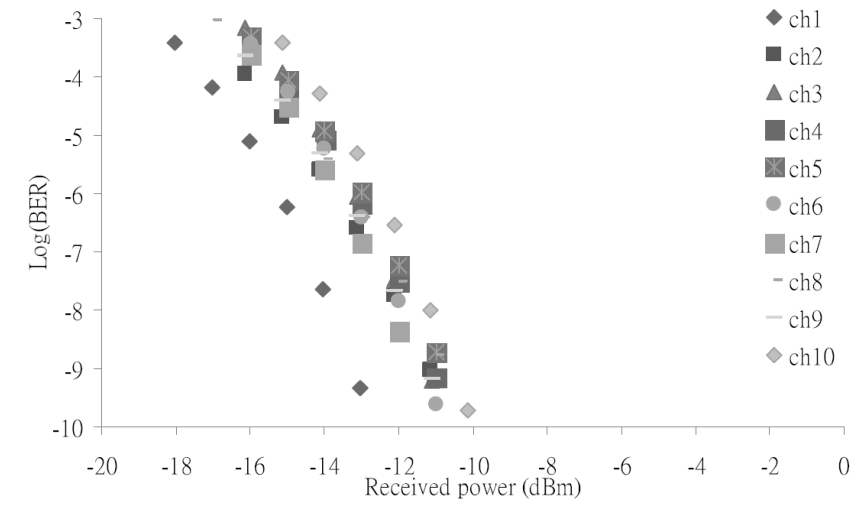

Fig. 14. BER curves of different individual SCM OFDM channels after transmission.

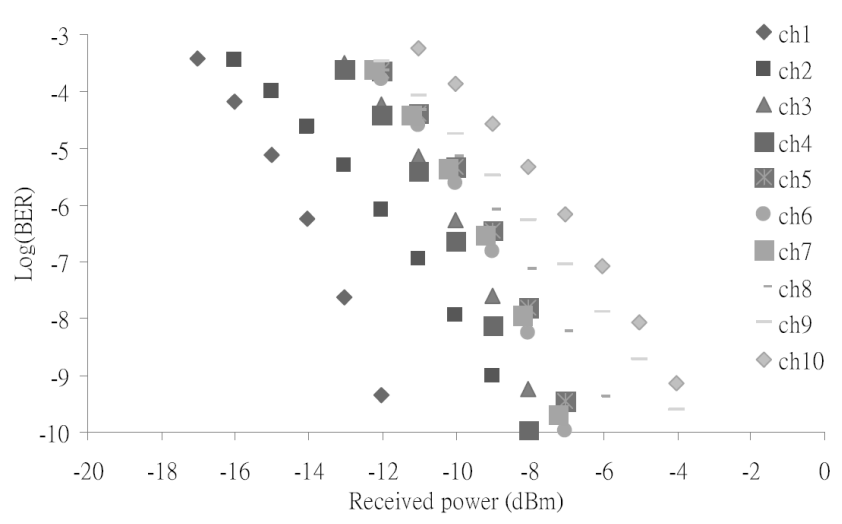

Fig. 15. BER curves of the $100 \mathrm{~Gb} / \mathrm{s}$ OFDM SCM signal after transmission.

fiber architecture. The architecture can significantly mitigate most of the RB noises, while maintaining the merits of using single distribution and drop fiber to reach the customer ONUs. Two different colorless RONU architectures: RSOA-based and FP-LD-based RONU, were tested and compared, showing that despite the polarization controlling issue, FP-LD can provide a greater downstream OFDM signal suppression and produce a better upstream data. Besides, a carrier distributed OFDMPON using wavelength-shifting has also been proposed to mitigate RB. In the heterogeneous optical wired/wireless access network based on WDM approach, negligible power penalty was observed in the $10 \mathrm{~Gb} / \mathrm{s} \mathrm{NRZ} \mathrm{signal} \mathrm{and} 1 \mathrm{~dB}$ power penalty was observed in the $10 \mathrm{~Gb} / \mathrm{s}$ OFDM ROF signal. Besides, we also propose an integrated wired/wireless network using $10 \mathrm{~Gb} / \mathrm{s} \mathrm{NRZ} \mathrm{signal} \mathrm{and} 10 \mathrm{~Gb} / \mathrm{s}$ OFDM on $60 \mathrm{GHz}$ carrier ROF signal. In the $100 \mathrm{~Gb} / \mathrm{s}$ OFDM-PON, numerical simulations were performed.

\section{REFERENCES}

[1] S. Chi, C. H. Yeh, C. W. Chow, "Broadband access technology for passive optical network," Proc. of SPIE, vol. 7234, 723408-1, 2008.

[2] J. Armstrong, "OFDM for Optical Communications," J. Lightw. Technol., vol. 27, no. 3, pp. 189-204, 2009.

[3] H. Takahashi, A. Al Amin, S. L. Jansen, I. Morita and H. Tanaka, "DWDM Transmission with 7.0-bit/s/Hz Spectral Efficiency using 8x65.1-Gbit/s Coherent PDM-OFDM Signals," Proc. OFC'09, San Diego, CA, USA, PDPB7.

[4] B. J. C. Schmidt, Z. Zan, L. B. Du and A. J. Lowery, "100 Gbit/s Transmission using Single-Band Direct-Detection Optical OFDM," Proc. OFC'09, San Diego, CA, USA, PDPC3. 
[5] T. Kobayashi, E. Yamazaki, E.Yamada, H. Masuda, A. Sano, E. Yoshida, Y. Miyamoto, K. Ishihara, R. Kudo, Y. Takatori, M. Mizoguchi, "Ultra long-haul transmission over $6,000 \mathrm{~km}$ of $100 \mathrm{~Gb} / \mathrm{s}$ serial signal by using coherent detection," Proc. OFC'09, San Diego, CA, USA, OWW4.

[6] Y. Tang, Y. Ma and W. Shieh, "107 Gb/s CO-OFDM Transmission with Inline Chromatic Dispersion Compensation," Proc. OFC'09, San Diego, CA, USA, OWW3.

[7] T. Duong, N. Genay, A. Pizzinat, B. Charbonnier, P. Chanclou, C. Kazmierski, "Low cost multi band-OFDM for remote modulation of colourless ONU in hybrid WDM/TDM-PON architecture," Proc. of ECOC, 2007, Berlin, Germany, Paper 5.4.2

[8] D. Qian, N. Cvijetic, J. Hu, T. Wang, "108 Gb/s OFDMA-PON with Polarization Multiplexing and Direct-Detection," Proc. OFC'09, San Diego, CA, USA PDPD5.

[9] C. W. Chow, C. H. Yeh, C. H. Wang, F. Y. Shih, and S. Chi "Rayleigh Backscattering Performance of OFDM-QAM in Carrier Distributed Passive Optical Networks," IEEE Photon. Technol. Lett., vol. 20, pp. $1848-1850,2008$

[10] C. W. Chow, C. H. Yeh, C. H. Wang, F. Y. Shih and S. Chi, "Signal remodulation of OFDM-QAM for long reach carrier distributed passive optical networks," IEEE Photon. Technol. Lett., vol. 21, pp. 715-717, 2009.

[11] M. -F. Huang, J. Yu, D. Qian, N. Cvijetic, and G. -K. Chang, "Lightwave Centralized WDM-OFDM-PON Network Employing Costeffective Directly Modulated Laser," Proc. OFC'09, San Diego, CA, USA, OMV5.

[12] A. Chowdhury, H. -C. Chien, M. -F. Huang, J. Yu, G. -K. Chang, "Rayleigh Backscattering Noise-Eliminated 115-km Long-Reach Bidirectional Centralized WDM-PON With 10-Gb/s DPSK Downstream and Remodulated 2.5-Gb/s OCS-SCM Upstream Signal," IEEE Photon. Technol. Lett., vol. 20, no. 24, pp. 2081-2083, 2008.

[13] L. Chen, J. G. Yu, S. Wen, J. Lu, Z. Dong, M. Huang, and G. -K. Chang, "A Novel Scheme for Seamless Integration of ROF With Centralized Lightwave OFDM-WDM-PON System," J. Lightw. Technol., vol. 27, pp. 2786-2791, 2009

[14] C. W. Chow, C. H. Yeh, Y. T. Li, C. H. Wang, F. Y. Shih, Y. M. Lin, C. L. Pan and S. Chi, "Demonstration of High Spectral Efficient Long Reach Passive Optical Networks using OFDM-QAM,' Proc. CLEO'08, San Jose, USA, CPDB7.

[15] C. W. Chow, C. H. Yeh, C. H. Wang, F. Y. Shih, C. L. Pan, and S. Chi, "WDM extended reach passive optical networks using OFDM-QAM," Opt. Express, vol. 16, pp. 12096-12101, 2008.

[16] G. K. Chang, J. Yu, Z. Jia, and J. Yu, "Novel optical-wireless access network architecture for simultaneously providing broadband wireless and wired services," Proc. OFC'06, Anaheim, CA, OFM1.

[17] D. Qian, J. Hu, P. N. Ji, T. Wang, "10.8-Gb/s OFDMA-PON Transmission Performance Study," Proc. OFC'09, San Diego, CA, USA, NME6.

[18] R. Dischler, F. Buchali, "Experimental Investigation of Non-Linear Threshold of $113 \mathrm{~Gb} / \mathrm{s}$ O-OFDM Signals on DCF Free Transmission Links," Proc. OFC'09, San Diego, CA, USA, OWW2.

[19] G. Talli, C. W. Chow, P. D. Townsend, "Modeling of modulation formats for interferometric noise mitigation," J. Lightw. Technol., vol. 26, pp. 3190-3198, 2008

[20] G. Talli, D. Cotter, P. D. Townsend, "Rayleigh backscattering impairments in access networks with centralised light source," Electron. Lett., vol. 42, pp. 877-878, 2006.

[21] L. Y. Chan, C. K. Chan, D. T. K. Tong, F. Tong, and L. K. Chen, "Upstream traffic transmitter using injection-locked Fabry-Perot laser diode as modulator for WDM access networks," Electron. Lett., vol. 38, pp. 43-45, 2002.

[22] W. Hung, C. K. Chan, L. K. Chen, and F. Tong, "An optical network unit for WDM access networks with downstream DPSK and upstream remodulated OOK data using injection-locked FP laser," IEEE Photon. Technol. Lett., vol. 15, pp. 1476-1478, 2003.

[23] C. W. Chow, "Wavelength remodulation using DPSK down-andupstream with high extinction ratio for 10-Gb/s DWDM-passive optical networks," IEEE Photon. Technol. Lett., vol. 20, pp.12-14, 2008.

[24] G. Talli, C. W. Chow, E. K. MacHale, and P. D. Townsend, "Long reach hybrid DWDM-TDM PON with high split ratio employing centralized light source," J. Optical Networking, vol. 6, pp. 765-776, 2007.

[25] C. W. Chow and H. K. Tsang, "Orthogonal Label Switching using Polarization-Shift-Keying Payload and Amplitude-Shift-Keying Label", IEEE Photon. Technol. Lett., vol. 17, pp. 2475-2477, 2005.

[26] L.Y. Chan, C.K. Chan, D.T.K Tong, E Tong and L.K. Chen, "Upstream traffic transmitter using injection-locked Fabry-Perot laser diode as modulator for WDM access networks," Electron. Lett., vol. 38, pp. 4345, 2002.
Chi-Wai Chow (S'02-M'04) received the B.Eng. (First-Class Hons) and $\mathrm{Ph} . \mathrm{D}$. degrees both from the Department of Electronic Engineering, the Chinese University of Hong Kong in 2001 and 2004 respectively. His Ph.D. focused on photonic packet switched networks. Then, he was a Postdoctoral Fellow at the Chinese University of Hong Kong, working on hybrid integration of photonic devices and silicon photonics. Between 2005-2007, he was a Postdoctoral Research Scientist in the Tyndall National Institute and Department of Physics, University College Cork (UCC) in Ireland, working mainly on two European Union Projects: PIEMAN and TRIUMPH. In 2007, he joined the Department of Photonics, National Chiao Tung University in Taiwan, as an Assistant Professor. His research interests are passive optical networks, radio-over-fiber and photonic signal processing.

C. H. Yeh received his $\mathrm{PhD}$ degree from the Institute of Electro-Optical Engineering, National Chiao Tung University, Taiwan in 2004. In 2004, he joined the Information and Communications Research Laboratories (ICL), Industrial Technology Research Institute (ITRI) in Taiwan, as a Researcher. In 2008, he was promoted as a Senior Researcher in ICL/ITRI. His research interests are optical fiber communications, fiber lasers, fiber amplifiers and wireless/fiber access network technologies.

C. H. Wang (S'09) received his Bachelor and Master degrees from the National Sun Yat-sen University and the National Chiao Tung University respectively. He is currently pursuing his $\mathrm{Ph} . \mathrm{D}$. degree in the Institute of Electro-Optical Engineering, National Chiao Tung University, Taiwan.

C. L. Wu received his BEng from the Department of Electrical Engineering, National Cheng Kung University, Taiwan, in 2008. He is purchasing his master degree in the Department of Photonics, National Chiao Tung University, Taiwan. His research interests are PON, and OFDM optical communication.

Sien Chi received the B.S.E.E. degree from National Taiwan University, Taipei, Taiwan, and the M.S.E.E. degree from National Chiao-Tung University, Hsinchu, Taiwan, in 1959 and 1961, respectively. He received the Ph.D. degree in electro-physics from Polytechnic Institute, Brooklyn, NY, in 1971. From 1971 to 2004, he was a Professor at National Chiao-Tung University. From 1998 to 2001, he was the Vice President of the National Chiao-Tung University. He is currently a Chair Professor at Yuan-Ze University, Chung Li, Taiwan. He is a Fellow of the Optical Society of America (OSA), His research interests are optical-fiber communications, fast and slow light, passive optical networks, and microwave photonics.

Chinlon Lin received his BSEE from National Taiwan University (NTU), MS from University of Illinois, Champaign-Urbana, and his Ph. D. from University of California, Berkeley. He joined AT\&T Bell Labs' Laser Sciences Research Department, Communication Sciences Research Division, Holmdel, NJ, in 1974. He was the inventor of the tunable infrared fiber Raman lasers, and both dispersion-shifted fibers (DSF) and dispersion-compensating fibers (DCF). In 1984 he was on leave from Bell Labs as a Visiting Guest Professor at the COM Center of the Tech. Univ. of Denmark. After the AT\&T divestiture, he joined Bellcore (Bell Communications Research) as Director of Broadband Lightwave Systems Research

From 2003 to 2007 he was with Chinese University of Hong Kong (CUHK) as Chair Professor of Photonics and Director of the Center for Advanced Research in Photonics, for both the Electronic Engineering and Information Engineering departments. Professor Lin is currently a Nanyang Professor and Director of Photonics Research Center at the School of EEE, Nanyang Technological University, in Singapore.

Professor Lin has published over 180 papers and international conference presentations, contributed several invited book chapters and holds 17 patents. He was an Associate Editor for IEEE Journal of Lightwave Technology and IEEE Photonics Technology Letters, and was on the TPC for OFC and ECOC. He has edited 2 books; the recent one on "Broadband Optical Access Networks and Fiber-to-the Home" published by Wiley in August 2006

He is a Fellow of both IEEE's LEOS (Lasers and Electro-Optical Society) and OSA (Optical Society of America) 